\title{
Aminoether-phenolato Precatalysts of Divalent Rare Earths and Alkaline Earths for the Mono and Double Hydrophosphination of Activated Alkenes
}

Ivan V. Basalov, ${ }^{\dagger}$ Bo Liu, ${ }^{\S}$ Thierry Roisnel,${ }^{\S}$ Anton V. Cherkasov, ${ }^{\dagger}$ Georgy K. Fukin, ${ }^{\dagger}$ JeanFrançois Carpentier, ${ }^{*} \S$ Yann Sarazin*, ${ }^{\star}$ and Alexander A. Trifonov* ${ }^{*} \dagger$

$\dagger$ G. A. Razuvaev Institute of Organometallic Chemistry of Russian Academy of Sciences, Tropinina 49, GSP-445, 603950 Nizhny Novgorod (Russia)

$\S$ Institut des Sciences Chimiques de Rennes, UMR 6226 CNRS - Université de Rennes 1, Organometallics: Materials and Catalysis group, Campus de Beaulieu, 35042 Rennes (France)

\section{Contents}

NMR data for $\left\{\mathrm{LO}^{\mathrm{NO} 2}\right\} \mathrm{Ca}\left\{\mathrm{CH}\left(\mathrm{SiMe}_{3}\right)_{2}\right\} \cdot \mathrm{THF}(7)$ and $\left\{\mathrm{LO}^{\mathrm{NO} 2}\right\} \mathrm{Sr}\left\{\mathrm{CH}\left(\mathrm{SiMe}_{3}\right)_{2}\right\}(\mathbf{8})$

NMR monitoring of substrate conversion in hydrophosphination reactions

Characterization of new phosphines

Table of crystallographic data for $\left\{\mathrm{LO}^{\mathrm{NO} 4}\right\} \mathrm{Yb}\left\{\mathrm{N}\left(\mathrm{SiMe}_{3}\right)_{2}\right\}(\mathbf{3})$ and 7 
Figure S1. ${ }^{1} \mathrm{H}$ NMR spectrum of $\left\{\mathrm{LO}^{\mathrm{NO} 2}\right\} \mathrm{Ca}\left\{\mathrm{CH}\left(\mathrm{SiMe}_{3}\right)_{2}\right\} \cdot \mathrm{THF}(7)$ in benzene- $d_{6}\left(25^{\circ} \mathrm{C}\right.$, $500.13 \mathrm{MHz}$ )

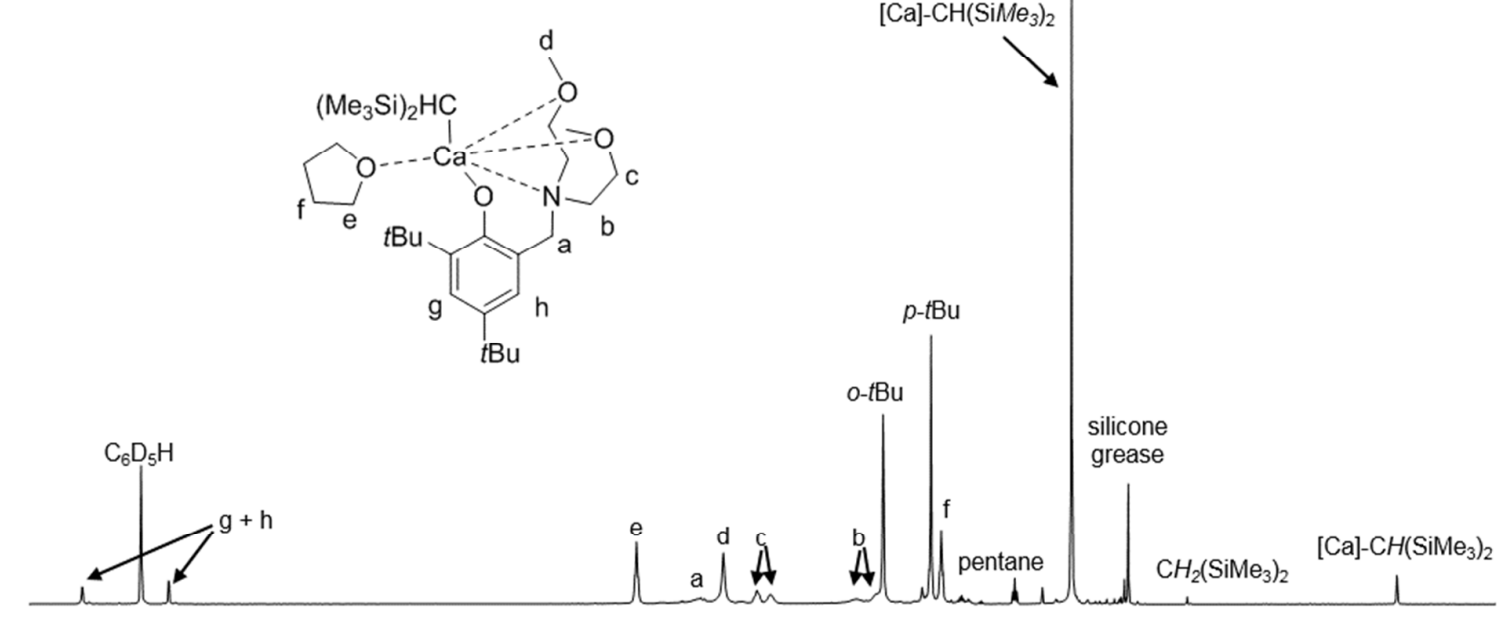

Figure S2. ${ }^{1} \mathrm{H}$ NMR spectrum of $\left\{\mathrm{LO}^{\mathrm{NO} 2}\right\} \mathrm{Sr}\left\{\mathrm{CH}\left(\mathrm{SiMe}_{3}\right)_{2}\right\}(\mathbf{8})$ in THF- $d_{8}\left(25^{\circ} \mathrm{C}, 500.13\right.$ $\mathrm{MHz}$ )
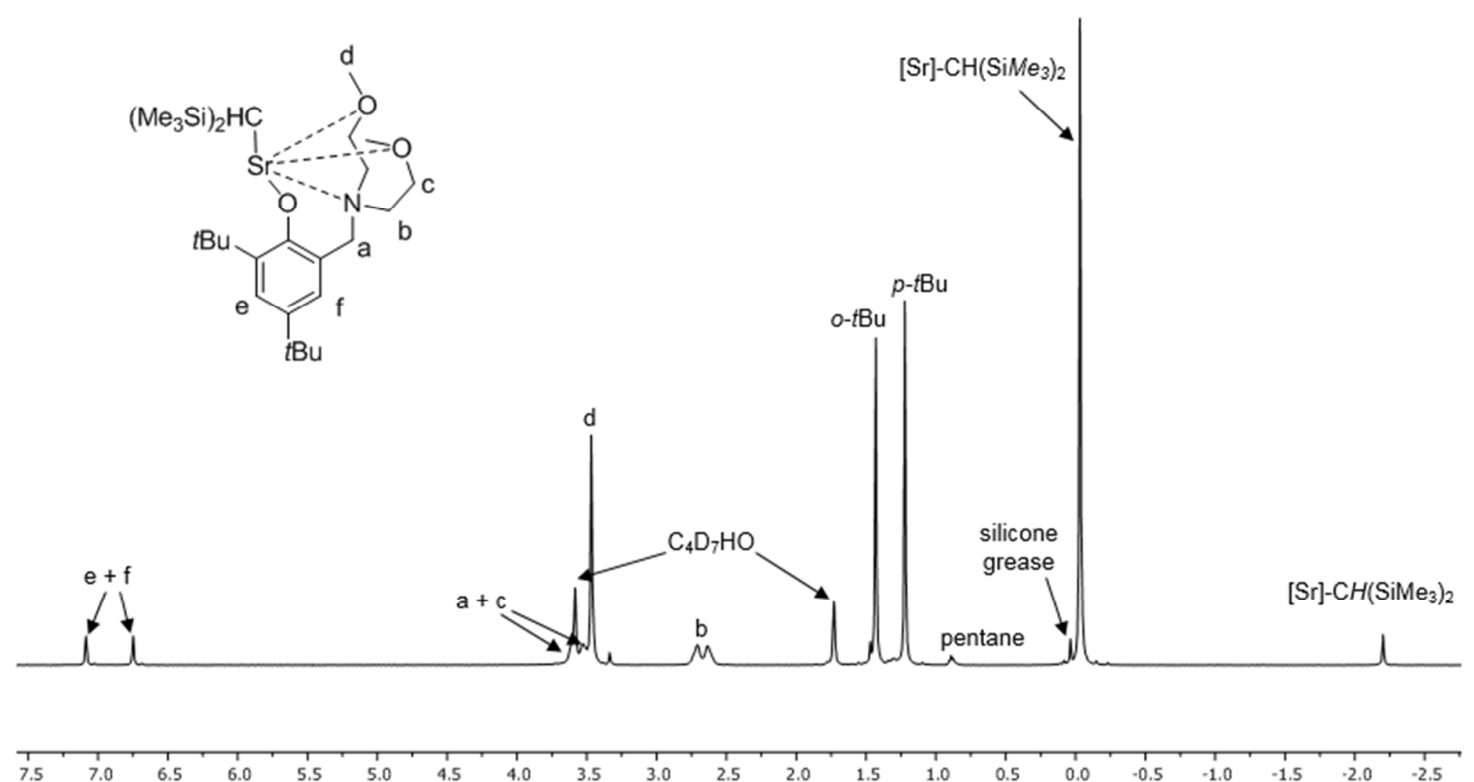
Figure S3. ${ }^{1} \mathrm{H}$ NMR spectrum $\left(500.13 \mathrm{MHz}\right.$, benzene- $\left.d_{6}, 25{ }^{\circ} \mathrm{C}\right)$ of the crude reaction mixture resulting from the double hydrophosphination of $p$ - $t \mathrm{Bu}$-styrene withPhPH $\mathrm{P}_{2}$ catalyzed by $\left\{\mathrm{LO}^{\mathrm{NO} 4}\right\} \mathrm{Yb}\left\{\mathrm{N}\left(\mathrm{SiMe}_{3}\right)_{2}\right\}$ (3) in benzene- $d_{6}$. $\left[\mathrm{PhPH}_{2}\right]_{0} /[p \text { - } t \text { Bu-styrene }]_{0} /[3]_{0}=25: 50: 1$; total volume $=0.6 \mathrm{~mL} ;[3]_{0}=72.2 \mathrm{mM}$.

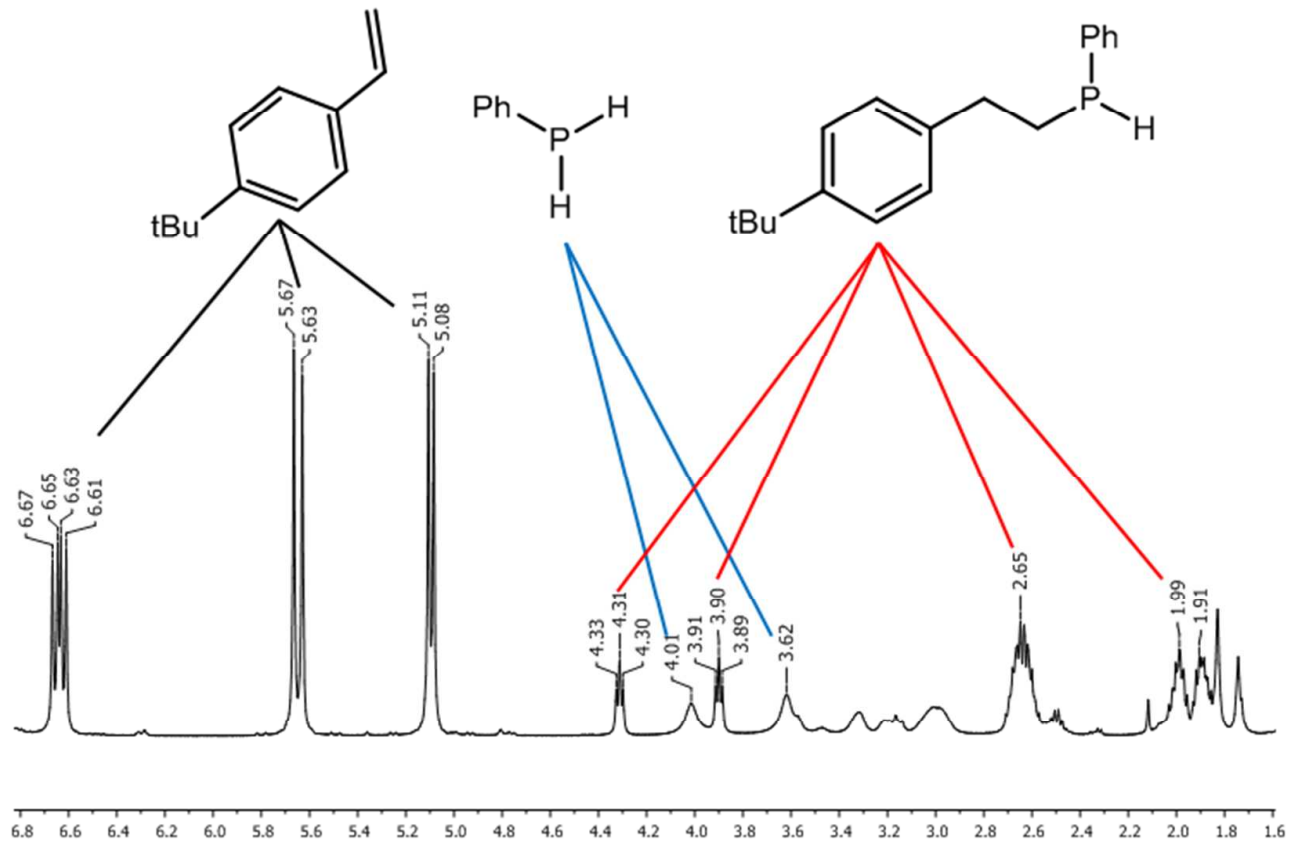

Figure S4. Details of the ${ }^{1} \mathrm{H}$ NMR spectrum $\left(500.13 \mathrm{MHz}\right.$, benzene- $d_{6}, 25{ }^{\circ} \mathrm{C}$ ) of the crude reaction mixture resulting from the hydrophosphination of $p$ - $t \mathrm{Bu}$-styrene withPhPH $\mathrm{PH}_{2}$ catalyzed by $\left\{\mathrm{LO}^{\mathrm{NO} 4}\right\} \mathrm{Yb}\left\{\mathrm{N}\left(\mathrm{SiMe}_{3}\right)_{2}\right\}$ (3) in benzene- $d_{6}$ at $25{ }^{\circ} \mathrm{C}$. $\left[\mathrm{PhPH}_{2}\right]_{0} /[p-t \mathrm{Bu} \text {-styrene }]_{0} /[3]_{0}=$ $25: 25: 1$; total volume $=0.6 \mathrm{~mL} ;[3]_{0}=36.0 \mathrm{mM}$.
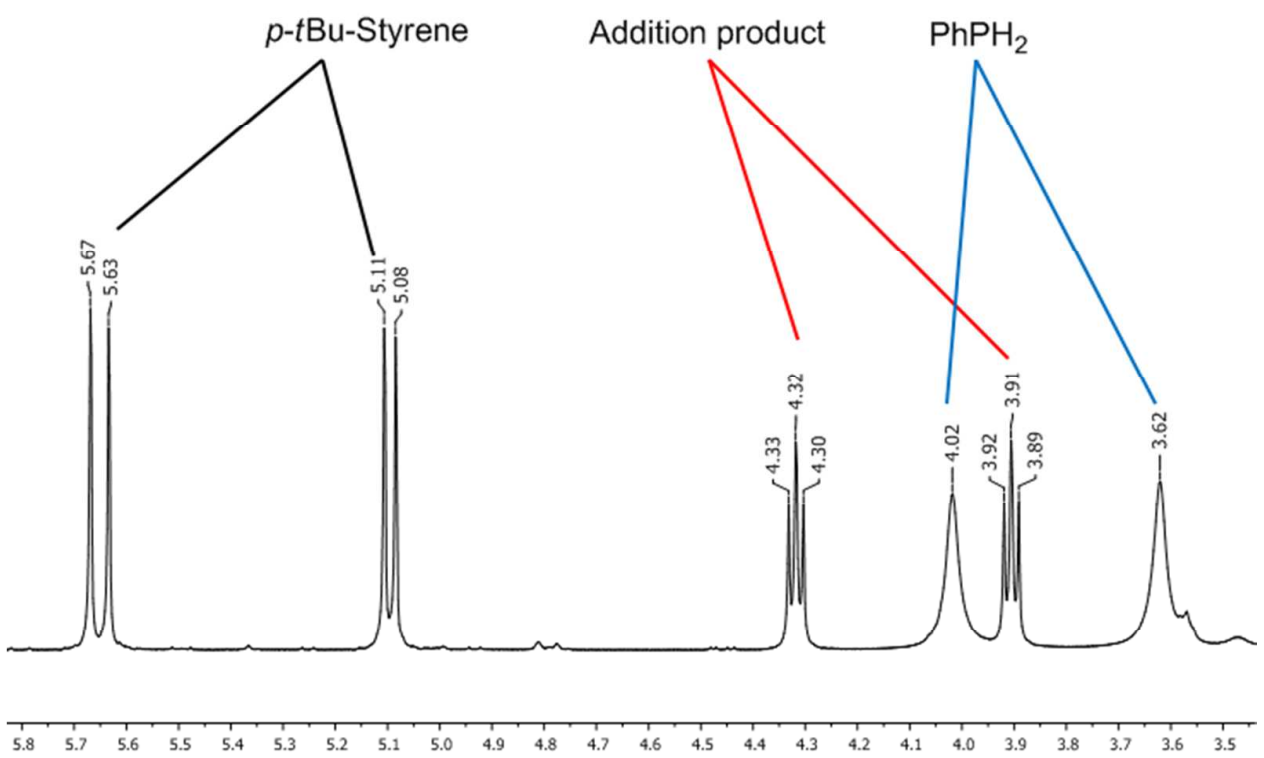
Figure S5. Detail of the ${ }^{1} \mathrm{H}$ NMR spectrum $\left(500.13 \mathrm{MHz}\right.$, benzene- $d_{6}, 25{ }^{\circ} \mathrm{C}$ ) of the crude reaction mixture resulting from the hydrophosphination of $p$-F-styrene with $\mathrm{PhPH}_{2}$ catalyzed by $\left\{\mathrm{LO}^{\mathrm{NO} 4}\right\} \mathrm{Yb}\left\{\mathrm{N}\left(\mathrm{SiMe}_{3}\right)_{2}\right\}$ (3) in benzene- $d_{6}$ at $25{ }^{\circ} \mathrm{C}$. $\left[\mathrm{PhPH}_{2}\right]_{0} /[p-\mathrm{F}-\text { styrene }]_{0} /[3]_{0}=$ 25:25:1; total volume $=0.6 \mathrm{~mL} ;[3]_{0}=36.0 \mathrm{mM}$.

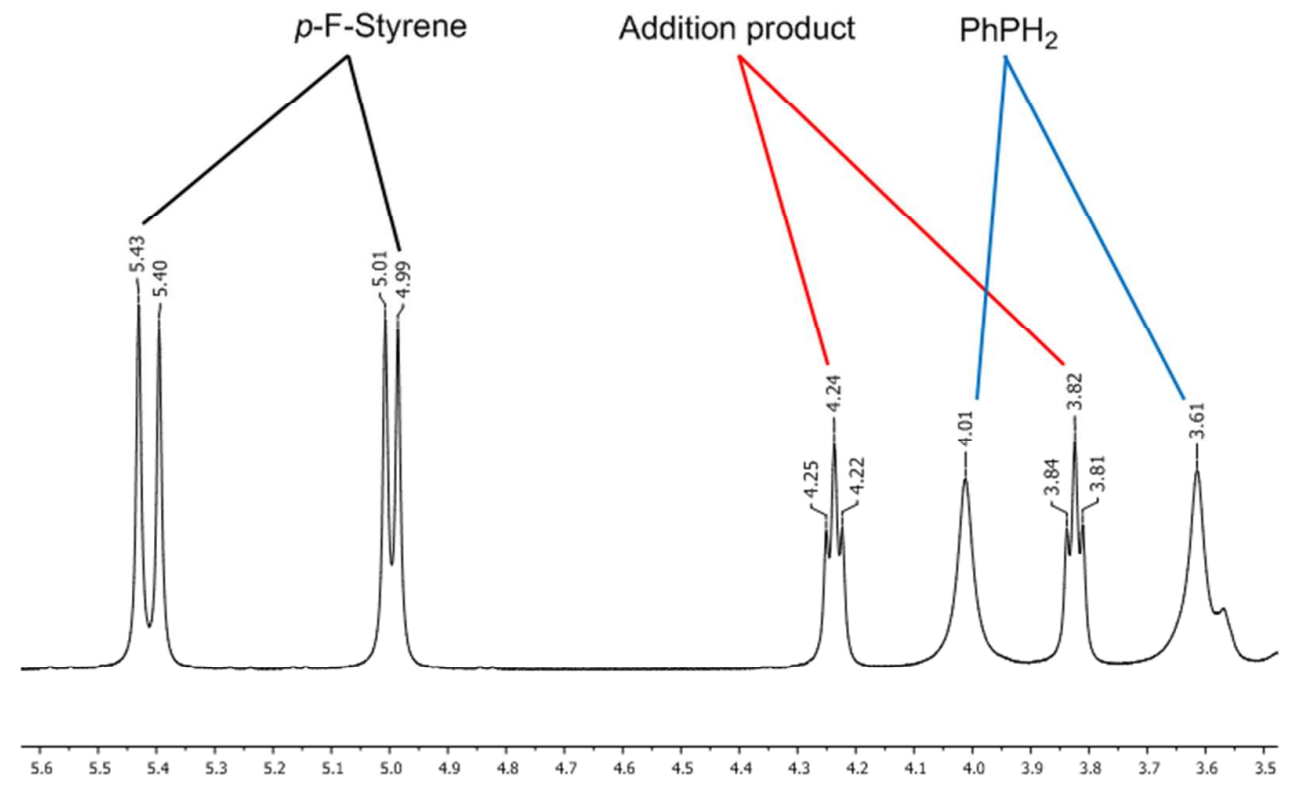

Figure S6. Detail of the ${ }^{1} \mathrm{H}$ NMR spectrum $\left(500.13 \mathrm{MHz}\right.$, benzene- $d_{6}, 25{ }^{\circ} \mathrm{C}$ ) of the crude reaction mixture resulting from the hydrophosphination of $p$-Cl-styrene with $\mathrm{PhPH}_{2}$ catalyzed by $\left\{\mathrm{LO}^{\mathrm{NO} 4}\right\} \mathrm{Yb}\left\{\mathrm{N}\left(\mathrm{SiMe}_{3}\right)_{2}\right\}$ (3) in benzene- $d_{6}$ at $25{ }^{\circ} \mathrm{C}$. $\left[\mathrm{PhPH}_{2}\right]_{0} /[p-\mathrm{Cl} \text {-styrene }]_{0} /[3]_{0}=$ $25: 25: 1$; total volume $=0.6 \mathrm{~mL} ;[3]_{0}=36.0 \mathrm{mM}$.

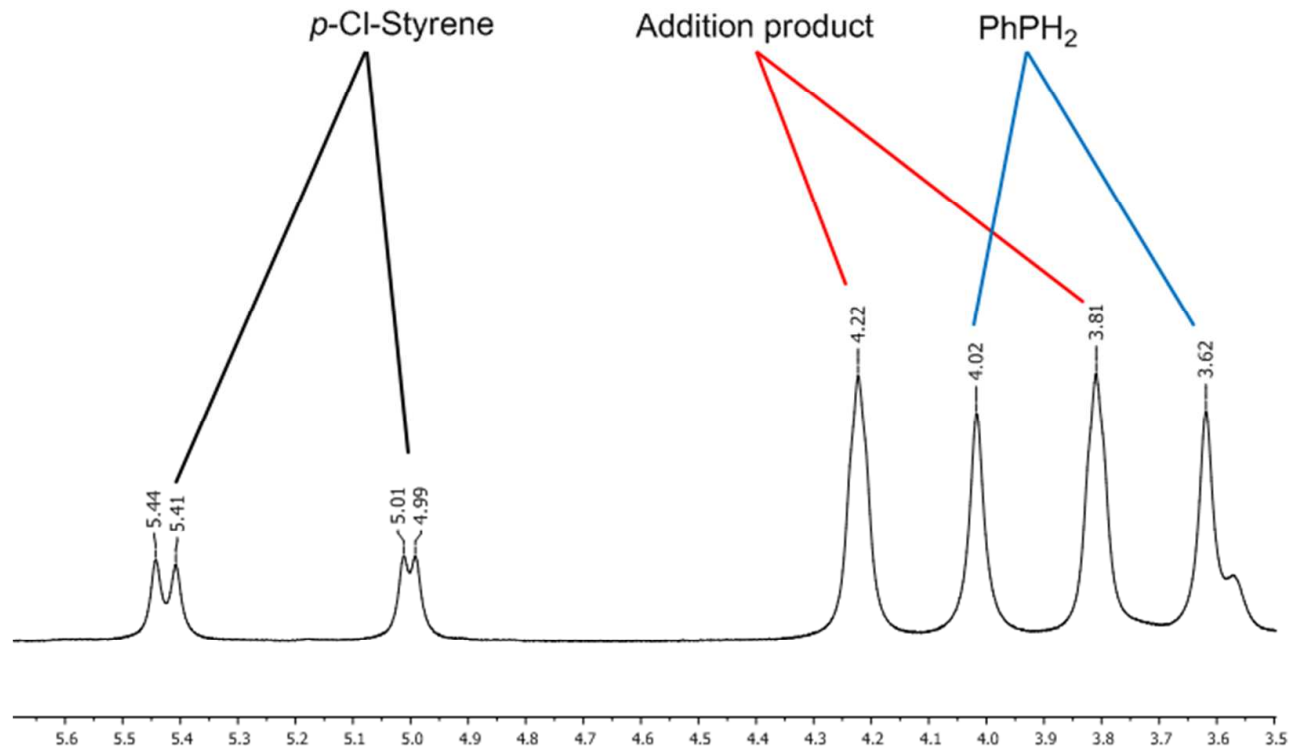


Figure S7. Detail of the ${ }^{1} \mathrm{H}$ NMR spectrum $\left(500.13 \mathrm{MHz}\right.$, benzene- $\left.d_{6}, 25{ }^{\circ} \mathrm{C}\right)$ of the crude reaction mixture resulting from the hydrophosphination of $p$-OMe-styrene withPhPH catalyzed by $\left\{\mathrm{LO}^{\mathrm{NO} 4}\right\} \mathrm{Yb}\left\{\mathrm{N}\left(\mathrm{SiMe}_{3}\right)_{2}\right\}$ (3) in benzene- $d_{6}$ at $25{ }^{\circ} \mathrm{C}$. $\left[\mathrm{PhPH}_{2}\right]_{0} /[p-\mathrm{OMe}-$ styrene $]_{0} /[3]_{0}=25: 25: 1$; total volume $=0.6 \mathrm{~mL} ;[3]_{0}=36.0 \mathrm{mM}$.

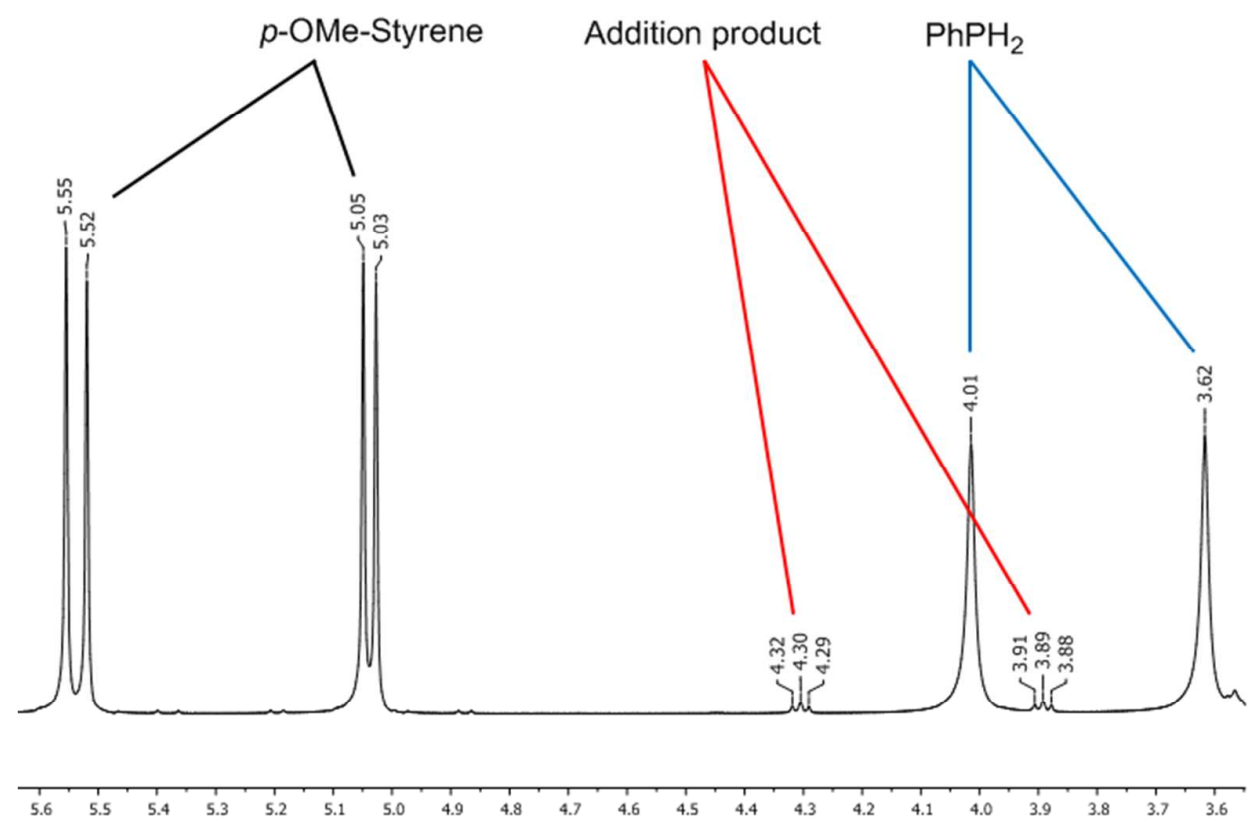

Figure S8. Detail of the ${ }^{1} \mathrm{H}$ NMR spectrum $\left(500.13 \mathrm{MHz}\right.$, benzene- $\left.d_{6}, 25{ }^{\circ} \mathrm{C}\right)$ of the crude reaction mixture resulting from the hydrophosphination of $p$-Me-styrene with $\mathrm{PhPH}_{2}$ catalyzed by $\left\{\mathrm{LO}^{\mathrm{NO} 4}\right\} \mathrm{Yb}\left\{\mathrm{N}\left(\mathrm{SiMe}_{3}\right)_{2}\right\} \quad$ (3) in benzene- $d_{6}$ at $25{ }^{\circ} \mathrm{C} . \quad\left[\mathrm{PhPH}_{2}\right]_{0} /[p-\mathrm{Me}-$ styrene $]_{0} /[3]_{0}=25: 25: 1$; total volume $=0.6 \mathrm{~mL} ;[3]_{0}=36.0 \mathrm{mM}$.

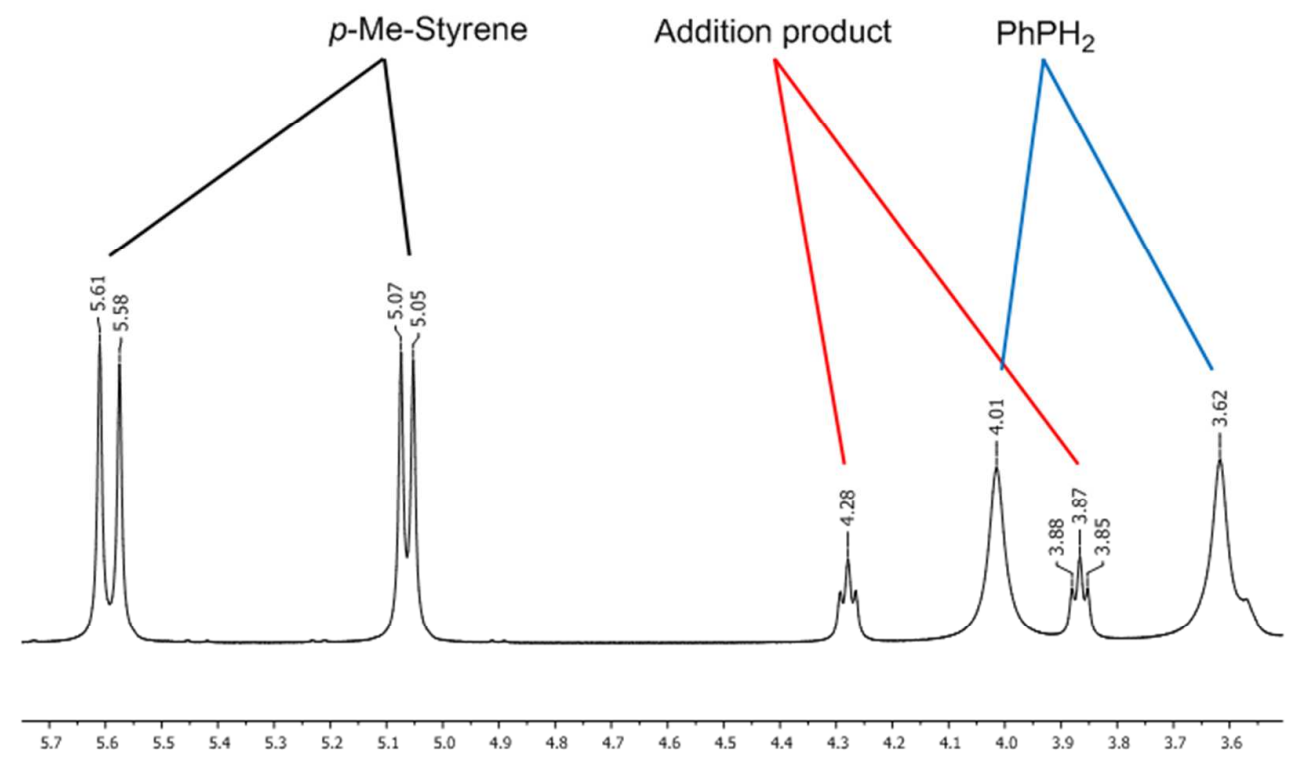


Figure S9. ${ }^{31} \mathrm{P}\left\{{ }^{1} \mathrm{H}\right\}$ NMR spectrum $\left(81 \mathrm{MHz}\right.$, benzene- $\left.d_{6}, 25{ }^{\circ} \mathrm{C}\right)$ of the product of double hydrophosphination reaction of styrene with $\mathrm{PhPH}_{2}$ catalyzed by $\left\{\mathrm{LO}^{\mathrm{NO} 4}\right\} \mathrm{Yb}\left\{\mathrm{N}\left(\mathrm{SiMe}_{3}\right)_{2}\right\}$ (3) in benzene- $d_{6}$ at $25{ }^{\circ} \mathrm{C}$. $\left[\mathrm{PhPH}_{2}\right]_{0} /[\text { styrene }]_{0} /[3]_{0}=25: 50: 1$; total volume $=0.6 \mathrm{~mL} ;[3]_{0}=36.0$ $\mathrm{mM}$.
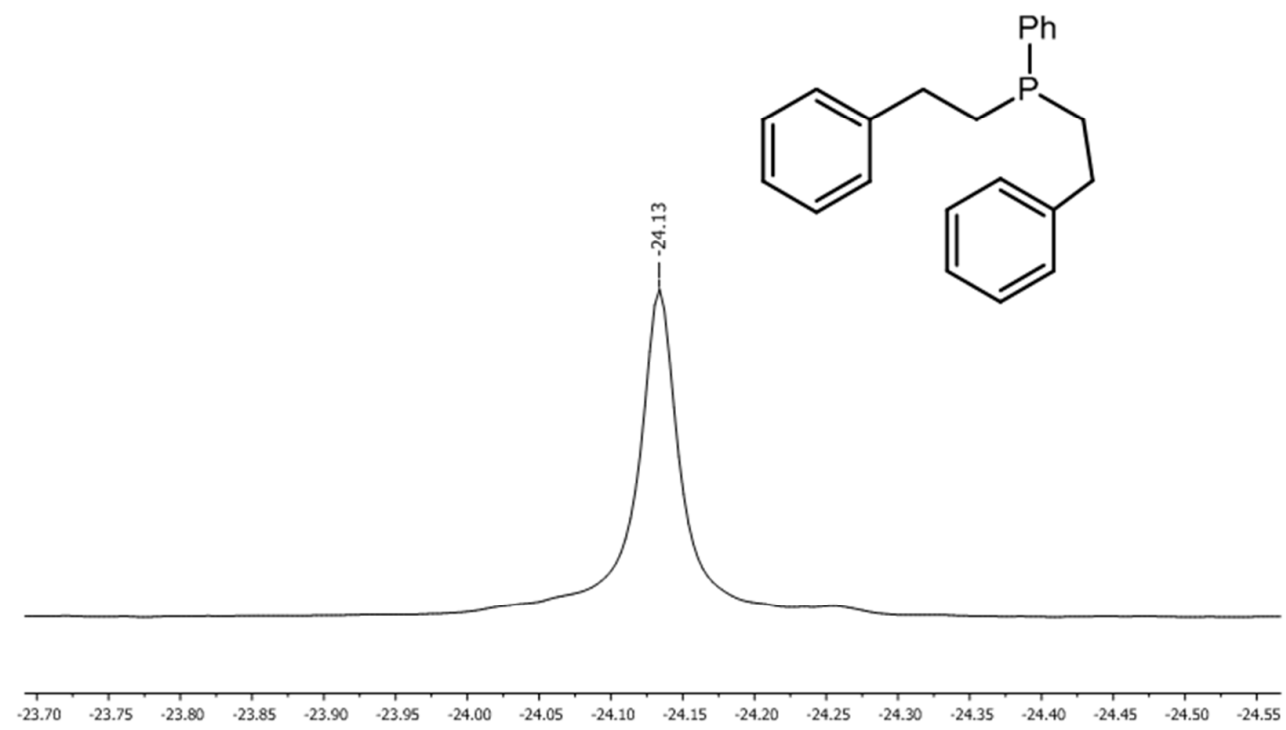

Figure S10. ${ }^{31} \mathrm{P}\left\{{ }^{1} \mathrm{H}\right\} \mathrm{NMR}$ spectrum $\left(81 \mathrm{MHz}, \mathrm{C}_{6} \mathrm{D}_{6}, 25{ }^{\circ} \mathrm{C}\right)$ of the product of the two-step reaction of $\mathrm{PhPH}_{2}$, styrene and $p$-Cl-styrene. First step: hydrophosphination of styrene with $\mathrm{PhPH}_{2}$ catalyzed by $\left\{\mathrm{LO}^{\mathrm{NO} 4}\right\} \mathrm{Yb}\left\{\mathrm{N}\left(\mathrm{SiMe}_{3}\right)_{2}\right\} \quad$ (3) in benzene- $d_{6}$ at $25{ }^{\circ} \mathrm{C}, 2 \mathrm{~h}$. $\left[\mathrm{PhPH}_{2}\right]_{0} /[\text { styrene }]_{0} /[3]_{0}=50: 50: 1 ;[3]_{0}=36.0 \mathrm{mM}$. Second step: $p$-Cl-styrene (50 equiv vs. 3 ) was then added to this reaction mixture, total volume $=0.6 \mathrm{~mL}$.
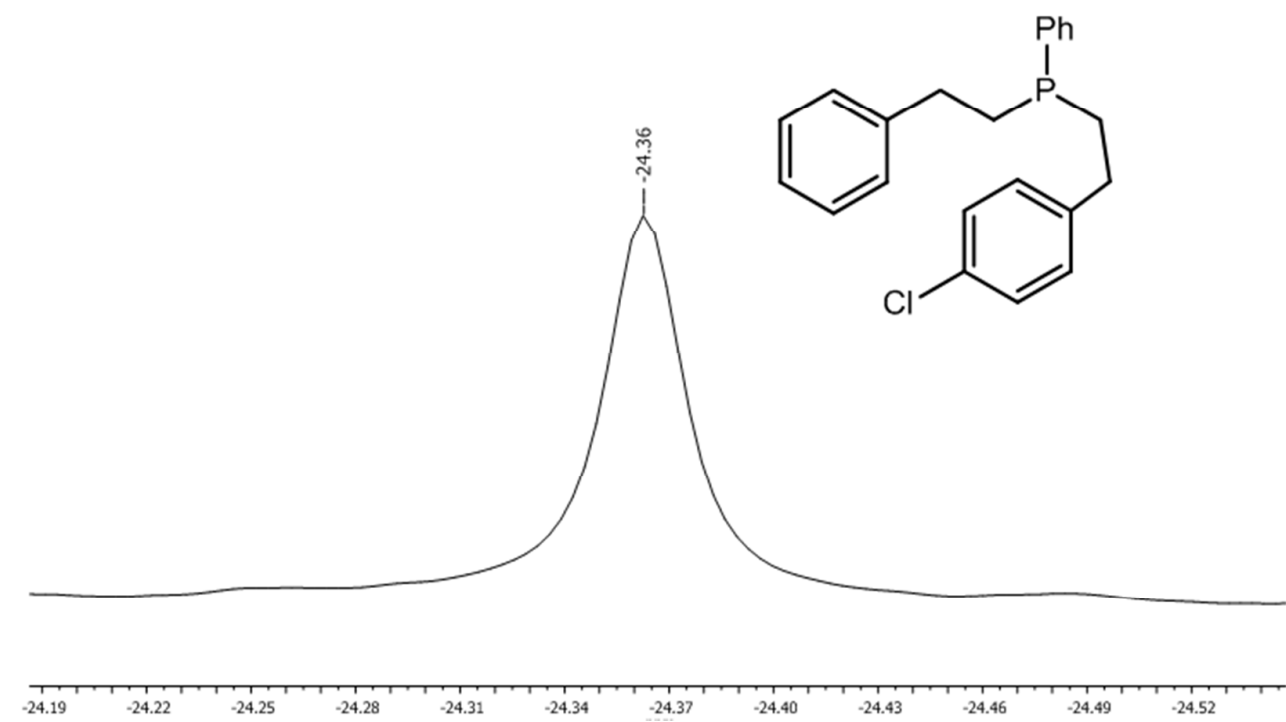
Figure S11. ${ }^{31} \mathrm{P}\left\{{ }^{1} \mathrm{H}\right\}$ NMR spectrum $\left(81 \mathrm{MHz}, \mathrm{C}_{6} \mathrm{D}_{6}, 25^{\circ} \mathrm{C}\right)$ of the product of the two-step reaction of $\mathrm{PhPH}_{2}$, styrene and $p$-F-styrene. First step: hydrophosphination of styrene with $\mathrm{PhPH}_{2}$ catalyzed by $\left\{\mathrm{LO}^{\mathrm{NO} 4}\right\} \mathrm{Yb}\left\{\mathrm{N}\left(\mathrm{SiMe}_{3}\right)_{2}\right\} \quad$ (3) in benzene- $d_{6}$ at $25{ }^{\circ} \mathrm{C}, 2 \mathrm{~h}$. $\left[\mathrm{PhPH}_{2}\right]_{0} /[\text { styrene }]_{0} /[3]_{0}=50: 50: 1 ;[3]_{0}=36.0 \mathrm{mM}$. Second step: $p$-F-styrene (50 equiv vs. 3) was then added to this reaction mixture, total volume $=0.6 \mathrm{~mL}$.

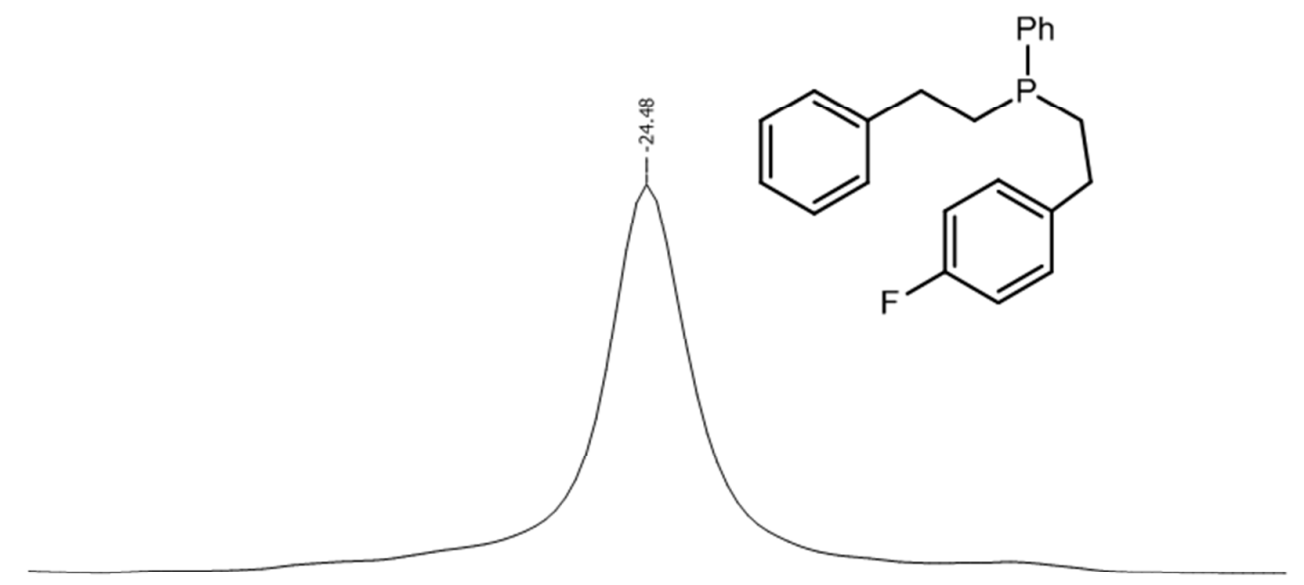

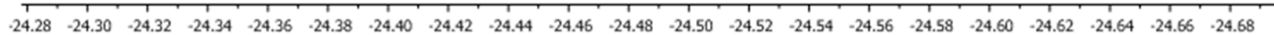


Figure S12. ${ }^{31} \mathrm{P}\left\{{ }^{1} \mathrm{H}\right\}$ NMR spectrum $\left(81 \mathrm{MHz}, \mathrm{C}_{6} \mathrm{D}_{6}, 25^{\circ} \mathrm{C}\right)$ of the early stage of the second step of the two-step reaction of $\mathrm{PhPH}_{2}$, styrene and $p$ - $t \mathrm{Bu}$-styrene. First step: hydrophosphination of styrene with $\mathrm{PhPH}_{2}$ catalyzed by $\left\{\mathrm{LO}^{\mathrm{NO} 4}\right\} \mathrm{Yb}\left\{\mathrm{N}\left(\mathrm{SiMe}_{3}\right)_{2}\right\}$ (3) in benzene- $d_{6}$ at $25{ }^{\circ} \mathrm{C}, 2 \mathrm{~h}$. $\left[\mathrm{PhPH}_{2}\right]_{0} /[\text { styrene }]_{0} /[3]_{0}=50: 50: 1 ;[3]_{0}=36.0 \mathrm{mM}$. Second step: $p$ $t$ Bu-styrene (50 equiv vs. 3) was then added to this reaction mixture, total volume $=0.6 \mathrm{~mL}$.

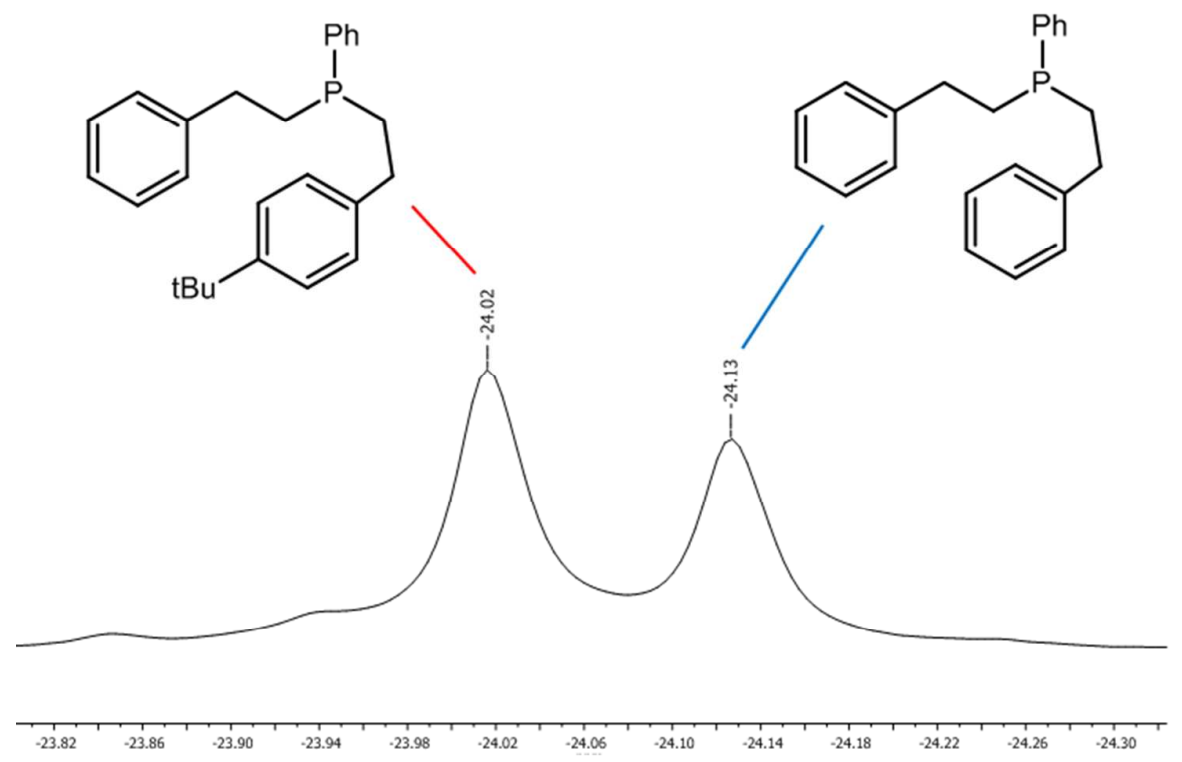

Figure S13. ${ }^{31} \mathrm{P}\left\{{ }^{1} \mathrm{H}\right\}$ NMR spectrum $\left(81 \mathrm{MHz}, \mathrm{C}_{6} \mathrm{D}_{6}, 25{ }^{\circ} \mathrm{C}\right)$ of the late stage of the two-step reaction of $\mathrm{PhPH}_{2}$, styrene and $p$ - $t \mathrm{Bu}$-styrene. First step: hydrophosphination of styrene with $\mathrm{PhPH}_{2}$ catalyzed by $\left\{\mathrm{LO}^{\mathrm{NO} 4}\right\} \mathrm{Yb}\left\{\mathrm{N}\left(\mathrm{SiMe}_{3}\right)_{2}\right\} \quad$ (3) in benzene- $d_{6}$ at $25{ }^{\circ} \mathrm{C}, 2 \mathrm{~h}$. $\left[\mathrm{PhPH}_{2}\right]_{0} /[\text { styrene }]_{0} /[3]_{0}=50: 50: 1 ;[3]_{0}=36.0 \mathrm{mM}$. Second step: $p$ - $t$ Bu-styrene (50 equiv vs. 3) was then added to this reaction mixture, total volume $=0.6 \mathrm{~mL}$.
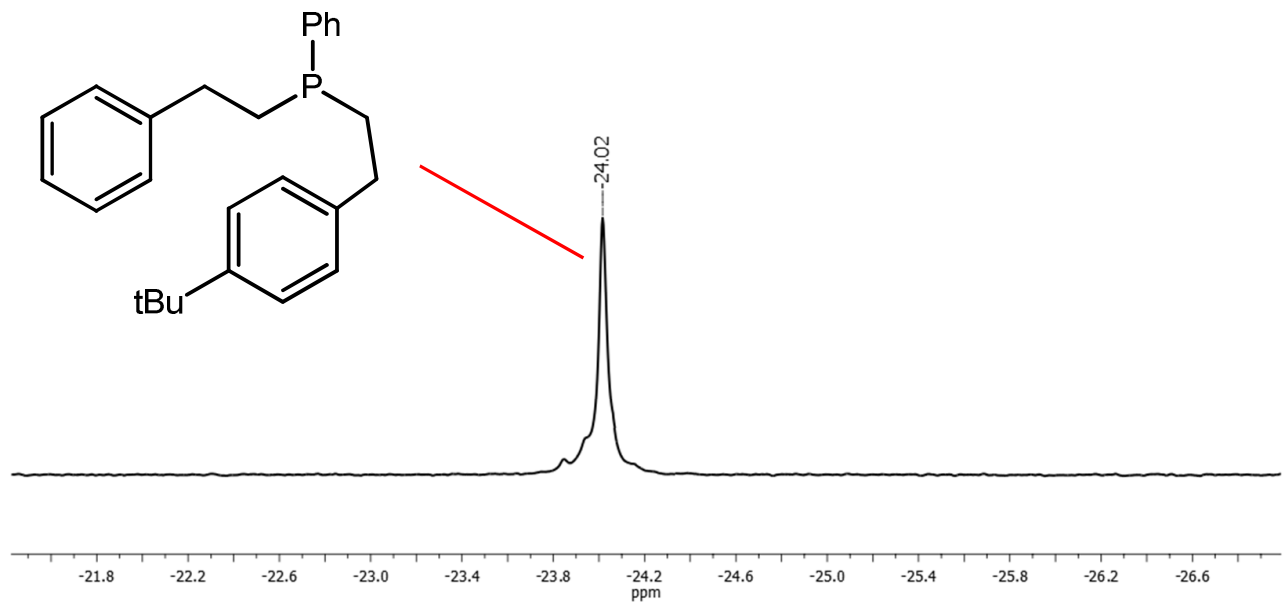
Figure S14. Characterization of $\mathrm{PhP}\left(\mathrm{CH}_{2} \mathrm{CH}_{2} \mathrm{Ph}\right)\left(\mathrm{CH}_{2} \mathrm{CH}_{2}-\mathrm{C}_{6} \mathrm{H}_{4}-p-\mathrm{F}\right)$<smiles>Fc1ccc(CCP(CCc2ccccc2)c2ccccc2)cc1</smiles>

${ }^{1} \mathrm{H}$ NMR $\left(200 \mathrm{MHz}, \mathrm{CDCl}_{3}, 298 \mathrm{~K}\right): \delta=1.67-1.95\left(\mathrm{~m}, 4 \mathrm{H}, \mathrm{CH}_{2}\right), 2.37-2.71\left(\mathrm{~m}, 4 \mathrm{H}, \mathrm{CH}_{2}\right)$, 6.66-6.82 (m, 4H, Ar-H), 6.94-7.22 (m, 8H, Ar-H), 7.40-7.53 (m, 2H, Ar-H) ppm.

${ }^{13} \mathrm{C}\left\{{ }^{1} \mathrm{H}\right\} \mathrm{NMR}\left(50 \mathrm{MHz}, \mathrm{CDCl}_{3}, 298 \mathrm{~K}\right): \delta=32.3\left(\mathrm{CH}_{2}\right), 32.6\left(\mathrm{CH}_{2}\right), 115.0\left(m-C_{6} \mathrm{H}_{4} \mathrm{~F}\right), 115.5$ $\left(m-C_{6} \mathrm{H}_{4} \mathrm{~F}\right), 132.5\left(i-\mathrm{C}_{6} \mathrm{H}_{4} \mathrm{~F}\right), 132.9\left(o-C_{6} \mathrm{H}_{4} \mathrm{~F}\right), 126.1,128.3,128.6,128.8,129.2,129.7$, $137.9,138.2,138.5$ (all aromatic $C) 142.7\left(i-C_{6} \mathrm{H}_{5}\right), 142.9\left(i-C_{6} \mathrm{H}_{5}\right), 159.0\left(p-C_{6} \mathrm{H}_{4} \mathrm{~F}\right) \mathrm{ppm}$.

${ }^{31} \mathrm{P}\left\{{ }^{1} \mathrm{H}\right\} \mathrm{NMR}\left(81 \mathrm{MHz}, \mathrm{CDCl}_{3}, 298 \mathrm{~K}\right): \delta=-24.48 \mathrm{ppm}$ (see Figure $\left.\mathrm{S} 11\right)$.

EI MS: $[\mathrm{M}+\mathrm{H}]^{+}, \mathrm{m} / \mathrm{z}$ calcd 337.15 , found 337.78 .

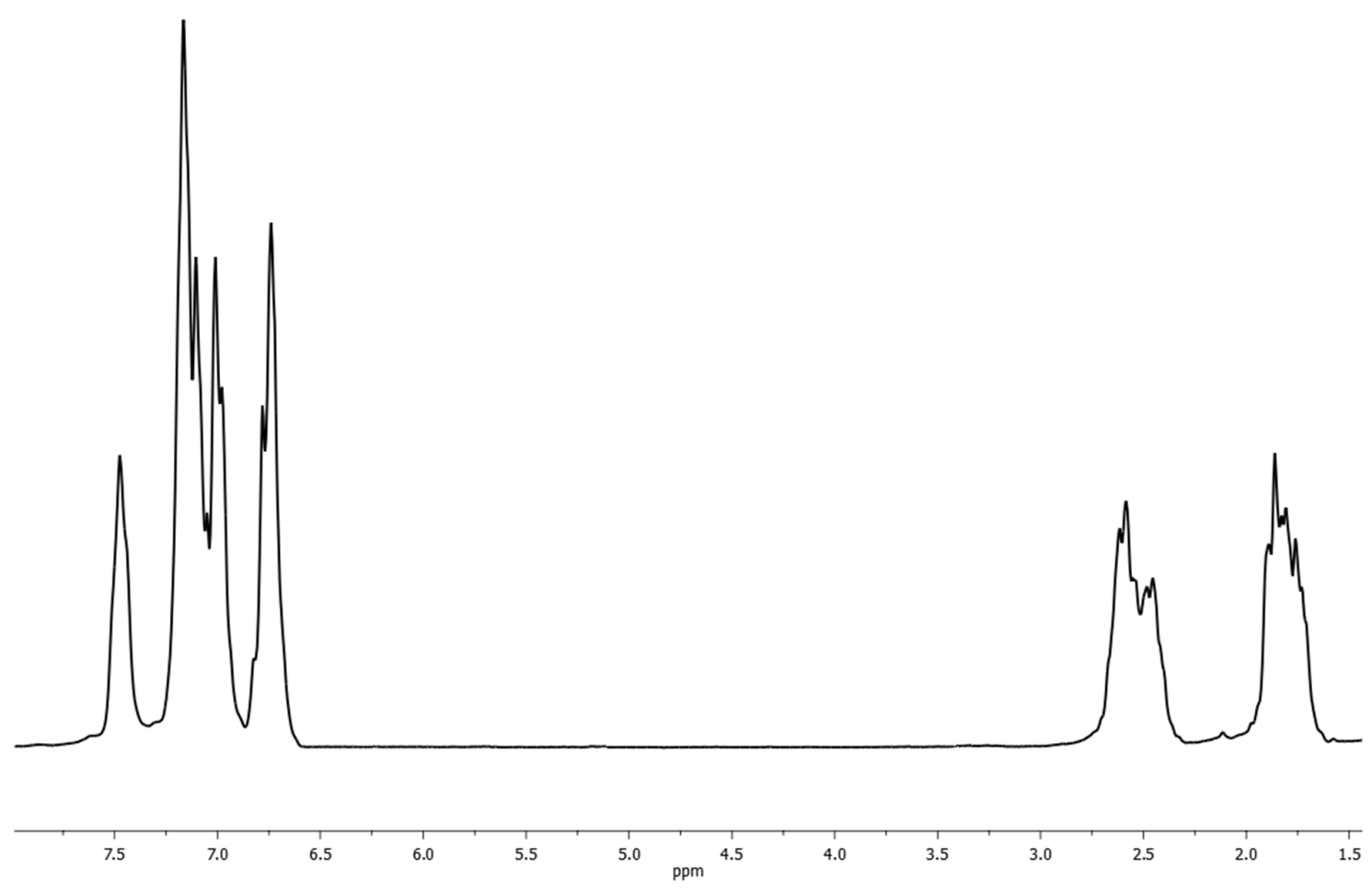

${ }^{1} \mathrm{H}$ NMR spectrum $\left(200 \mathrm{MHz}, \mathrm{CDCl}_{3}, 298 \mathrm{~K}\right)$ of $\mathrm{PhP}\left(\mathrm{CH}_{2} \mathrm{CH}_{2} \mathrm{Ph}\right)\left(\mathrm{CH}_{2} \mathrm{CH}_{2}-\mathrm{C}_{6} \mathrm{H}_{4}-p-\mathrm{F}\right)$. 
Figure S15. Characterization of $\mathrm{PhP}\left(\mathrm{CH}_{2} \mathrm{CH}_{2} \mathrm{Ph}\right)\left(\mathrm{CH}_{2} \mathrm{CH}_{2}-\mathrm{C}_{6} \mathrm{H}_{4}-p-\mathrm{Cl}\right)$<smiles>Clc1ccc(CCP(CCc2ccccc2)c2ccccc2)cc1</smiles>

${ }^{1} \mathrm{H}$ NMR $\left(200 \mathrm{MHz}, \mathrm{CDCl}_{3}, 298 \mathrm{~K}\right): \delta=1.80-1.94\left(\mathrm{~m}, 4 \mathrm{H}, \mathrm{CH}_{2}\right), 2.50-2.70\left(\mathrm{~m}, 4 \mathrm{H}, \mathrm{CH}_{2}\right)$, 6.60-6.72 (m, 4H, Ar-H), 6.94-7.24 (m, 8H, Ar-H), 7.40-7.53 (m, 2H, Ar-H) ppm.

${ }^{13} \mathrm{C}$ NMR $\left(50 \mathrm{MHz}, \mathrm{CDCl}_{3}, 298 \mathrm{~K}\right): \delta=32.3\left(\mathrm{CH}_{2}\right), 32.6\left(\mathrm{CH}_{2}\right), 126.0,127.3,127.8,128.2$, 128.4, 128.6, 128.9 (all arom- $C), 129.6\left(p-C_{6} \mathrm{H}_{4} \mathrm{Cl}\right), 131.6\left(i-C_{6} \mathrm{H}_{5}\right), 132.4\left(i-C_{6} \mathrm{H}_{5}\right), 132.8(i-$ $\left.\mathrm{C}_{6} \mathrm{H}_{4} \mathrm{Cl}\right) \mathrm{ppm}$.

${ }^{31} \mathrm{P}\left\{{ }^{1} \mathrm{H}\right\} \mathrm{NMR}\left(81 \mathrm{MHz}, \mathrm{CDCl}_{3}, 298 \mathrm{~K}\right): \delta=-24.36 \mathrm{ppm}$ (see Figure $\left.\mathrm{S} 10\right)$.

EI MS: $[\mathrm{M}+\mathrm{H}]^{+}, \mathrm{m} / \mathrm{z}$ calcd 353.12 , found 353.72 .
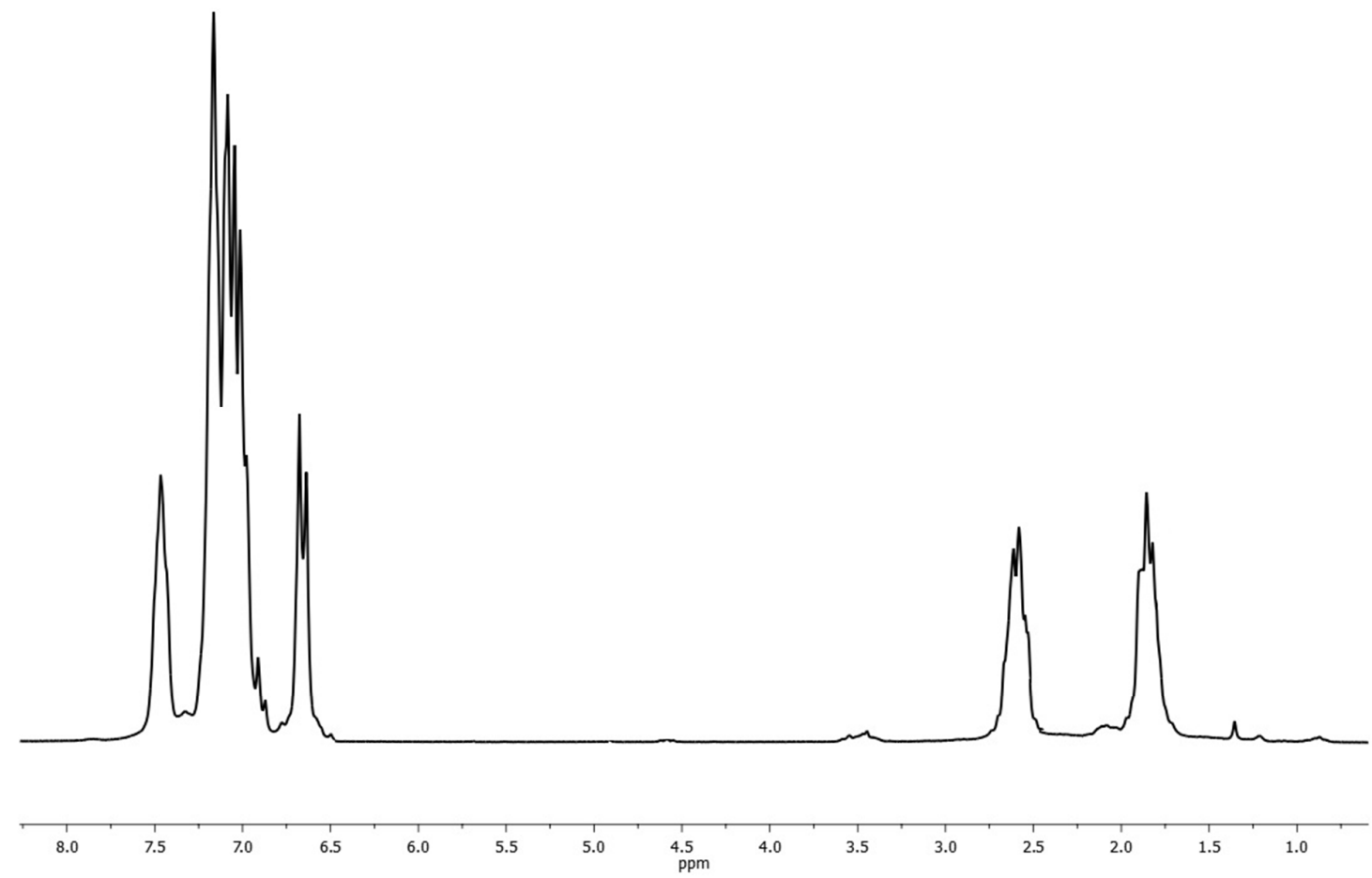

${ }^{1} \mathrm{H}$ NMR spectrum $\left(200 \mathrm{MHz}, \mathrm{CDCl}_{3}, 298 \mathrm{~K}\right)$ of $\mathrm{PhP}\left(\mathrm{CH}_{2} \mathrm{CH}_{2} \mathrm{Ph}\right)\left(\mathrm{CH}_{2} \mathrm{CH}_{2}-\mathrm{C}_{6} \mathrm{H}_{4}-p-\mathrm{Cl}\right)$. 
Figure S16. Characterization of $\mathrm{PhP}\left(\mathrm{CH}_{2} \mathrm{CH}_{2} \mathrm{Ph}\right)\left(\mathrm{CH}_{2} \mathrm{CH}_{2}-\mathrm{C}_{6} \mathrm{H}_{4}-p-{ }^{t} \mathrm{Bu}\right)$<smiles>CC(C)(C)c1ccc(CCP(CCc2ccccc2)c2ccccc2)cc1</smiles>

${ }^{1} \mathrm{HNMR}\left(200 \mathrm{MHz}, \mathrm{CDCl}_{3}, 298 \mathrm{~K}\right): \delta=1.23\left(\mathrm{~s}, 9 \mathrm{H}, \mathrm{C}\left(\mathrm{CH}_{3}\right)_{3}\right), 1.78-2.00\left(\mathrm{~m}, 4 \mathrm{H}, \mathrm{CH}_{2}\right), 2.50-$ 2.75 (m, 4H, $\mathrm{CH}_{2}$ ), 6.94-7.34 (m, 12H, Ar- $H$ ), 7.43-7.56 (m, 2H, Ar- $H$ ) ppm.

${ }^{13} \mathrm{C}$ NMR $\left(50 \mathrm{MHz}, \mathrm{CDCl}_{3}, 298 \mathrm{~K}\right): \delta=31.3\left(\mathrm{C}\left(\mathrm{CH}_{3}\right)_{3}\right), 32.3\left(\mathrm{CH}_{2}\right), 32.6\left(\mathrm{CH}_{2}\right), 34.1$ $\left(C\left(\mathrm{CH}_{3}\right)_{3}\right), 125.23,125.38,125.87,126.16,127.74,127.99,128.21,128.35,128.50,128.72$, 132.77 (all arom- $C), 133.7\left(i-C_{6} \mathrm{H}_{5}\right), 136.9\left(i-C_{6} \mathrm{H}_{5}\right), 148.4\left(i-C_{6} \mathrm{H}_{4}-{ }^{\mathrm{t}} \mathrm{Bu}\right), 150.5\left(p-C_{6} \mathrm{H}_{4}{ }^{\mathrm{t}} \mathrm{Bu}\right)$.

${ }^{31} \mathrm{P}\left\{{ }^{1} \mathrm{H}\right\}$ NMR (81 MHz, $\left.\mathrm{CDCl}_{3}, 298 \mathrm{~K}\right): \delta=-24.02 \mathrm{ppm}($ see Figure $\mathrm{S} 13)$.

EI MS: $[\mathrm{M}+\mathrm{H}]^{+}, \mathrm{m} / \mathrm{z}$ calcd 375.23 , found 375.81 .

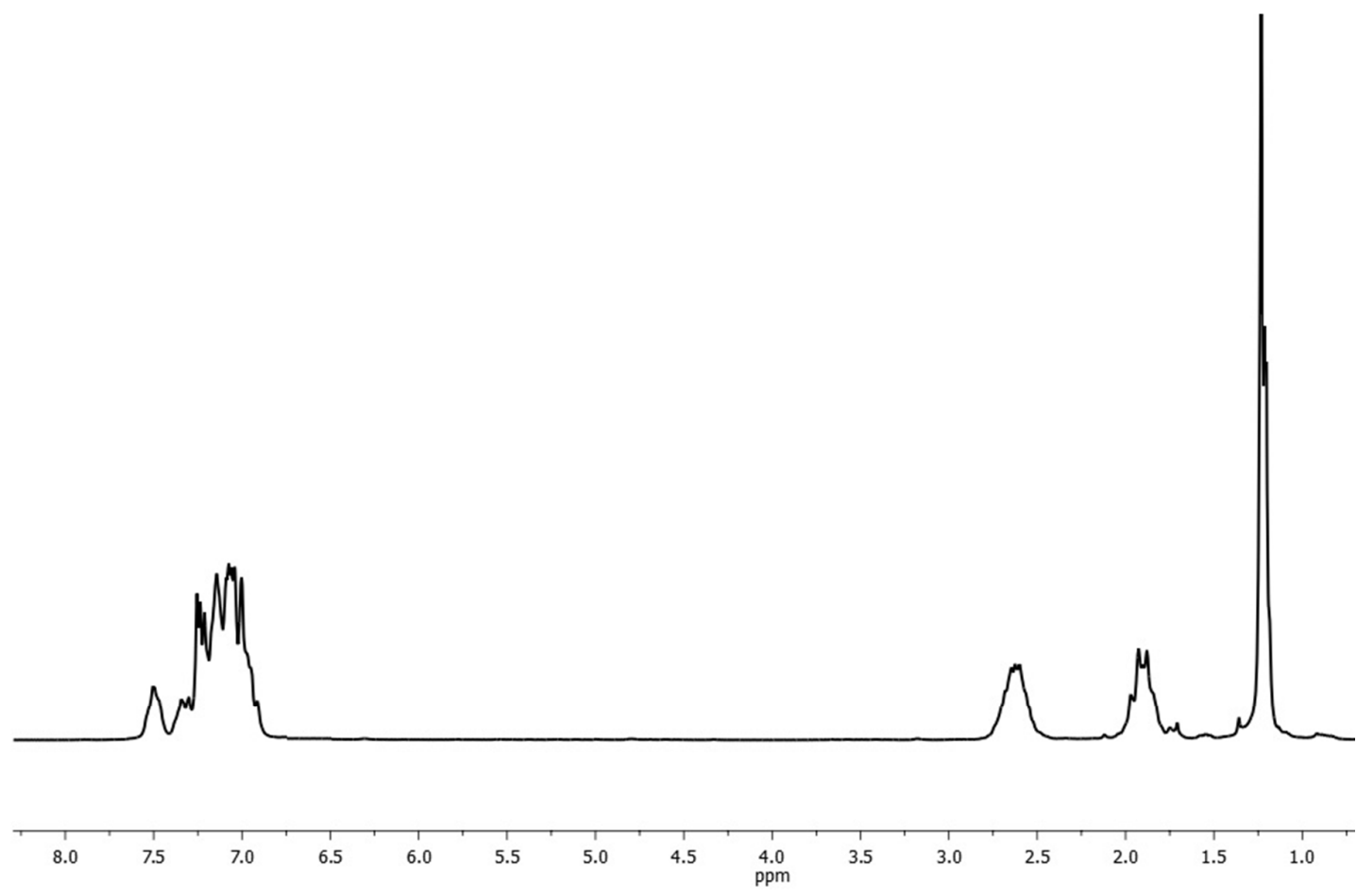

${ }^{1} \mathrm{H}$ NMR spectrum $\left(200 \mathrm{MHz}, \mathrm{CDCl}_{3}, 298 \mathrm{~K}\right)$ of $\mathrm{PhP}\left(\mathrm{CH}_{2} \mathrm{CH}_{2} \mathrm{Ph}\right)\left(\mathrm{CH}_{2} \mathrm{CH}_{2}-\mathrm{C}_{6} \mathrm{H}_{4}-p{ }^{\mathrm{t}} \mathrm{Bu}\right)$. 
Figure S17. Characterization of $\mathrm{HPPh}\left(\mathrm{CH}_{2} \mathrm{CH}_{2}-\mathrm{C}_{6} \mathrm{H}_{4}-p-\mathrm{Br}\right)$

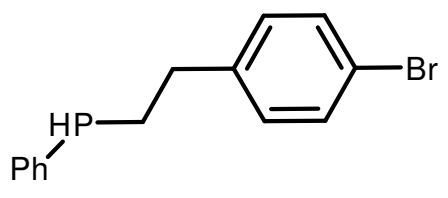

${ }^{1} \mathrm{HNMR}\left(200 \mathrm{MHz}, \mathrm{C}_{6} \mathrm{D}_{6}, 298 \mathrm{~K}\right): \delta=1.63-1.85\left(\mathrm{~m}, 2 \mathrm{H}, \mathrm{CH}_{2}\right), 2.34-2.51\left(\mathrm{~m}, 2 \mathrm{H}, \mathrm{CH}_{2}\right), 4,02$ $\left(\mathrm{dt},{ }^{1} J_{\mathrm{PH}}=206 \mathrm{~Hz},{ }^{3} J_{\mathrm{HH}}=6.9 \mathrm{~Hz}, 1 \mathrm{H}, \mathrm{PH}\right), 6.48-6.66(\mathrm{~m}, 2 \mathrm{H}, \mathrm{Ar}-H), 6.77-6.88(\mathrm{~m}, 1 \mathrm{H}, \mathrm{Ar}-$ $H)$, 6.98-7.34 (m, 6H, Ar-H) ppm.

${ }^{13} \mathrm{C}\left\{{ }^{1} \mathrm{H}\right\}$ NMR $\left(50 \mathrm{MHz}, \mathrm{C}_{6} \mathrm{D}_{6}, 298 \mathrm{~K}\right): \delta=\delta 24.97\left(\mathrm{CH}_{2}\right), 33.82\left(\mathrm{CH}_{2}\right), 114.18,119.81$, 127.27, 127.75, 128.23, 128.39, 129.93, 131.38, 133.43, 141.07 (all Ar-C) ppm.

${ }^{31} \mathrm{P}\left\{{ }^{1} \mathrm{H}\right\}$ NMR $\left(81 \mathrm{MHz}, \mathrm{C}_{6} \mathrm{D}_{6}, 298 \mathrm{~K}\right): \delta=-52.88 \mathrm{ppm}$.

EI MS: $[\mathrm{M}+\mathrm{H}]^{+}, \mathrm{m} / \mathrm{z}$ calcd 294.00, found 293.77.

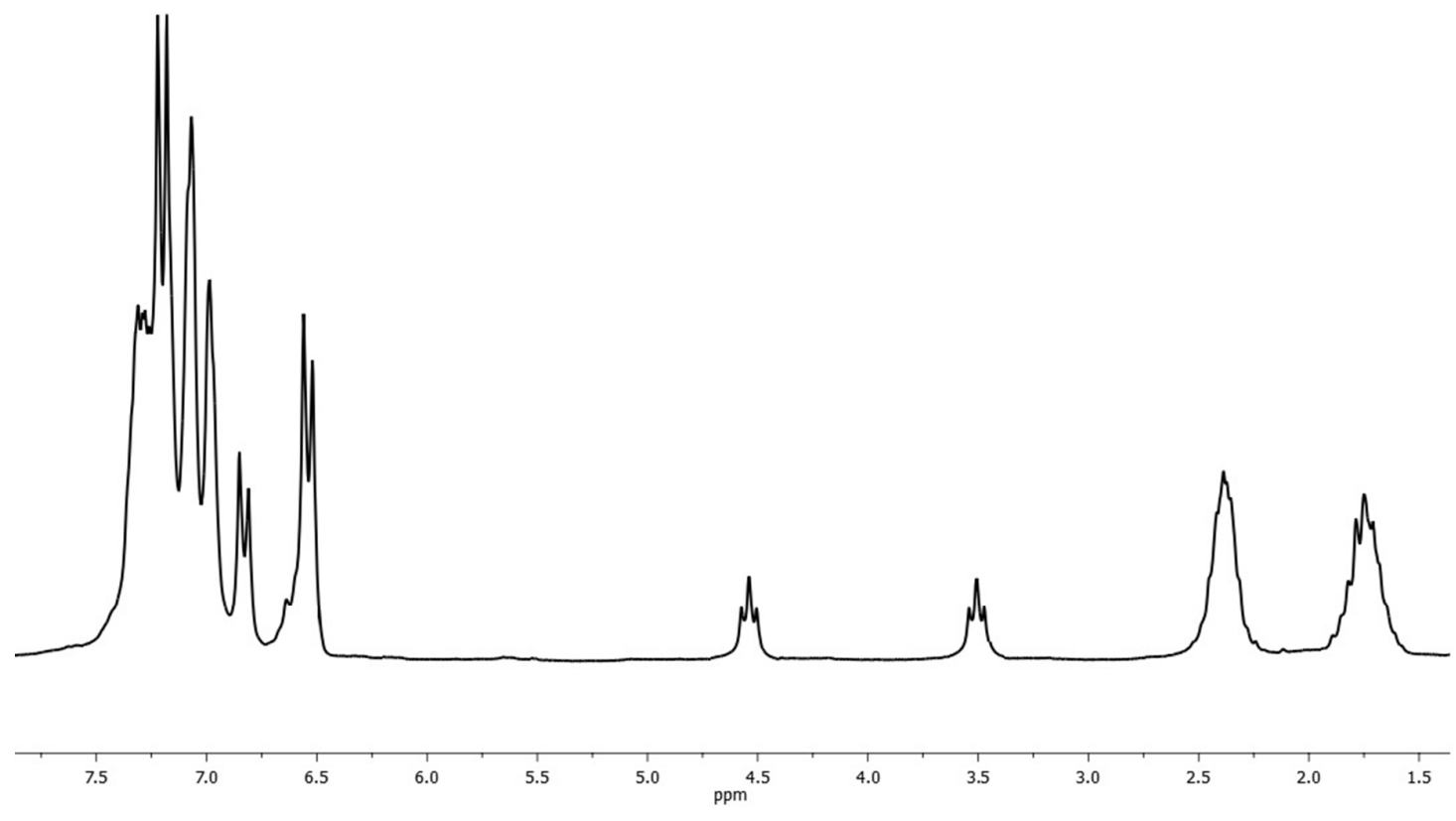

${ }^{1} \mathrm{H}$ NMR spectrum (200 MHz, $\mathrm{C}_{6} \mathrm{D}_{6}, 298 \mathrm{~K}$ ) of $\mathrm{HPPh}\left(\mathrm{CH}_{2} \mathrm{CH}_{2}-\mathrm{C}_{6} \mathrm{H}_{4}-p-\mathrm{Br}\right)$. 
Figure S18. Characterization of $\mathrm{HPPh}\left(\mathrm{CH}_{2} \mathrm{CH}_{2}-\mathrm{C}_{6} \mathrm{H}_{4}-m-\mathrm{OMe}\right)$

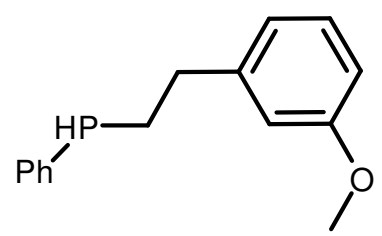

${ }^{1} \mathrm{HNMR}\left(200 \mathrm{MHz}, \mathrm{C}_{6} \mathrm{D}_{6}, 298 \mathrm{~K}\right): \delta=1.76-2.02\left(\mathrm{~m}, 2 \mathrm{H}, \mathrm{CH}_{2}\right), 2.48-2.70\left(\mathrm{~m}, 2 \mathrm{H}, \mathrm{CH}_{2}\right), 4,07$ $\left(\mathrm{dt},{ }^{1} J_{\mathrm{PH}}=207 \mathrm{~Hz},{ }^{3} J_{\mathrm{HH}}=6.9 \mathrm{~Hz}, 1 \mathrm{H}, \mathrm{PH}\right), 6.55-6.74(\mathrm{~m}, 3 \mathrm{H}, \mathrm{Ar}-H), 6.90-7.18(\mathrm{~m}, 4 \mathrm{H}, \mathrm{Ar}-$ $H), 7.22-7.42(\mathrm{~m}, 2 \mathrm{H}, \mathrm{Ar}-H) \mathrm{ppm}$.

${ }^{13} \mathrm{C}\left\{{ }^{1} \mathrm{H}\right\}$ NMR $\left(50 \mathrm{MHz}, \mathrm{C}_{6} \mathrm{D}_{6}, 298 \mathrm{~K}\right): \delta=25.23\left(\mathrm{CH}_{2}\right), 34.64\left(\mathrm{CH}_{2}\right), 54.41\left(\mathrm{OCH}_{3}\right), 111.49$, $114.16,120.57,128.02$, 128.33, 129.34, 133.71, 134.81, 135.83, 137.06, 143.82, 160.09 (all Ar- $C$ ).

${ }^{31} \mathrm{P}\left\{{ }^{1} \mathrm{H}\right\}$ NMR $\left(81 \mathrm{MHz}, \mathrm{C}_{6} \mathrm{D}_{6}, 298 \mathrm{~K}\right): \delta=-52.86$.

EI HRMS: $[\mathrm{M}+\mathrm{H}]^{+}, \mathrm{m} / \mathrm{z}$ calcd 244.10 , found 243.04 .

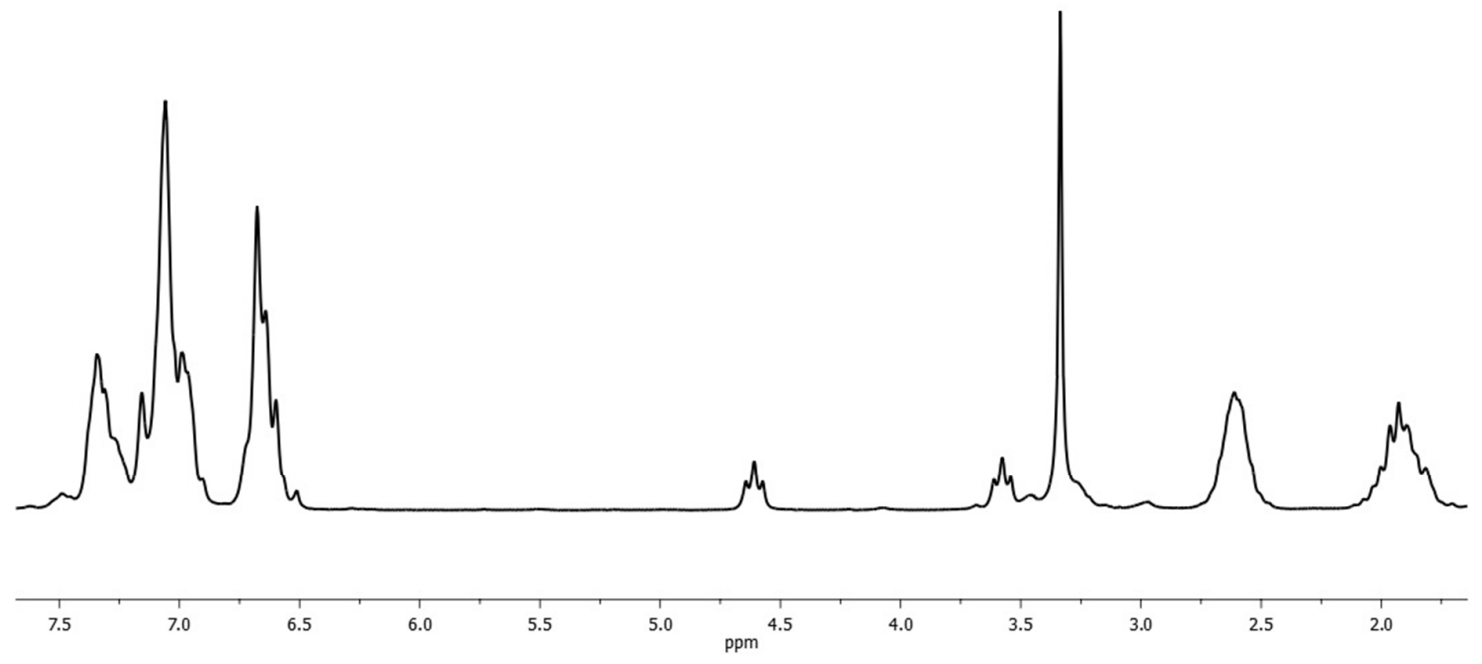

${ }^{1} \mathrm{H}$ NMR spectrum (200 MHz, $\left.\mathrm{C}_{6} \mathrm{D}_{6}, 298 \mathrm{~K}\right)$ of $\mathrm{HPPh}\left(\mathrm{CH}_{2} \mathrm{CH}_{2}-\mathrm{C}_{6} \mathrm{H}_{4}-m-\mathrm{OMe}\right)$. 
Table S1. Table of crystallographic data for $\mathbf{3}$ and $\mathbf{7 .}$

\begin{tabular}{|c|c|c|}
\hline & $\begin{array}{c}\left\{\mathrm{LO}^{\mathrm{NO} 4}\right\} \mathrm{Yb}\left(\mathrm{N}\left(\mathrm{SiMe}_{3}\right)_{2}\right) \\
(\mathbf{3})\end{array}$ & $\begin{array}{c}\left\{\mathrm{LO}^{\mathrm{NO} 2}\right\} \mathrm{Ca}\left\{\mathrm{CH}\left(\mathrm{SiMe}_{3}\right)_{2}\right\} \bullet \mathrm{THF} \\
(\mathbf{7})\end{array}$ \\
\hline Formula & $\mathrm{C}_{31} \mathrm{H}_{60} \mathrm{~N}_{2} \mathrm{O}_{5} \mathrm{Si}_{2} \mathrm{Yb}$ & $\mathrm{C}_{32} \mathrm{H}_{63} \mathrm{CaNO}_{4} \mathrm{Si}_{2}$ \\
\hline CCDC & 1045763 & 1468793 \\
\hline Mol. weight & 770.03 & 622.09 \\
\hline Crystal system & Monoclinic & Monoclinic \\
\hline Space group & $\mathrm{P} 21 / \mathrm{c}$ & $\mathrm{P} 21 / \mathrm{n}$ \\
\hline$a(\AA)$ & $11.52832(16)$ & $15.3747(8)$ \\
\hline$b(\AA)$ & $18.6070(2)$ & $10.6909(6)$ \\
\hline$c(\AA)$ & $17.9724(3)$ & $23.2876(14)$ \\
\hline$\alpha\left(^{0}\right)$ & 90 & 90 \\
\hline$\beta\left(\left(^{\circ}\right)\right.$ & $106.3560(15)$ & $96.413(3)$ \\
\hline$\gamma\left({ }^{\mathrm{o}}\right)$ & 90 & 90 \\
\hline $\mathrm{V}\left(\AA^{3}\right)$ & $3699.18(8)$ & $3803.8(4)$ \\
\hline$Z$ & 4 & 4 \\
\hline Density $\left(\mathrm{g} / \mathrm{cm}^{3}\right)$ & 1.383 & 1.086 \\
\hline Abs. coeff., $\left(\mathrm{mm}^{-1}\right)$ & 2.629 & 0.259 \\
\hline$F(000)$ & 1592 & 1368 \\
\hline Crystal size, mm & $0.20 \times 0.20 \times 0.20$ & $0.2 \times 0.15 \times 0.12$ \\
\hline \multirow[t]{2}{*}{$\theta$ range, deg } & 3.22 to 26.00 & 3.41 to 27.43 \\
\hline & $-14<h<14$ & $-19<h<19$ \\
\hline \multirow[t]{2}{*}{ Limiting indices } & $-22<k<22$ & $-13<k<12$ \\
\hline & $-22<l<22$ & $-29<l<30$ \\
\hline$R$ (int) & 0.0529 & 0.1056 \\
\hline Reflections collected & 56137 & 31000 \\
\hline Reflec. Unique $[\mathrm{I}>2 \sigma]$ & 7163 & 8620 \\
\hline Completeness to $\theta(\%)$ & 98.5 & 99.5 \\
\hline Data/restraints/para. & 7163 / 0 / 382 & $8620 / 0$ / 375 \\
\hline Goodness-of-fit & 1.175 & 1.028 \\
\hline$R_{1}[\mathrm{I}>2 \sigma]$ (all data) & $0.0319(0.0358)$ & $0.0763(0.1617)$ \\
\hline $\mathrm{w} R_{2}[\mathrm{I}>2 \sigma]$ (all data) & $0.0712(0.0725)$ & $0.1725(0.2070)$ \\
\hline Largest diff. e $\cdot \mathrm{A}^{-3}$ & 1.807 and -1.208 & 0.700 and -0.345 \\
\hline
\end{tabular}

